\title{
Energy-Efficient Cross-Layer Optimization for Wireless Sensor Networks
}

\author{
Yongqiang Fei, Peng Zhang, Yuping Zhao \\ School of Electronics Engineering and Computer Science, Peking University, Beijing, China \\ Email: fyq@pku.edu.cn, zhang_p@pku.edu.cn, yuping.zhao@pku.edu.cn
}

Received May 2013

\begin{abstract}
In this paper, we propose a cross-layer design combining adaptive modulation coding (AMC) and automatic repeat request (ARQ) to minimize the bit energy consumption under both packet loss rate and retransmission delay constraints. We analyze the best constellation size of M-ary square quadrature amplitude modulation (MQAM) in different distance, and give advice on retransmission limits under different packet loss rates. The impacts of path loss fading and additive white Gaussian noise (AWGN) are taken into consideration. The computation of energy consumption includes the circuit, transmission and retransmission energies at both transmitter and receiver sides. Numerical results are obtained to verify the validity of our design. We also show that the retransmission benefit varies with the packet loss rate constraint.
\end{abstract}

Keywords: Cross-Layer Design; Energy Consumption; Green Modulation; AMC; ARQ

\section{Introduction}

Along with the increasing demand for green communication, researches on energy efficiency have been prospered in recent decades, especially in wireless communication networks. Take sensor network for example, a single chip integrating baseband and radio frequency (RF) circuits may have to work with a small battery for years, whose battery replacement or charging, if possible, is difficult and expensive. Such scenarios bring high requirement of minimizing the energy consumption.

Among them, to find the energy efficient modulation type and constellation size is the central problem [1-4]. It is widely accepted that when transmission distance is far, transmission energy is dominant in total energy consumption, and small constellation size is more energy efficient. When transmission distance is short, circuit energy becomes comparable to transmission energy, resulting in an advantage of large constellation size.

At the same time, great interests are attracted by crosslayer design. By employing adaptive modulation coding (AMC) at physical layer and automatic repeat request (ARQ) or Hybrid ARQ (HARQ) at data link layer, the system could achieve a considerable gain in spectral efficiency or reduce the packet loss rate [5-8]. This is due to the fact that by adopting retransmission protocol to guarantee the information transmission, cross-layer design could allow for higher and more efficient transmission mode.

So far, few researches have discussed the cross-layer optimization on energy consumption. In [1], Cui et al. presented the RF transceiver circuit structure, discussed energy efficiency of coded M-ary square quadrature amplitude modulation (MQAM) and Multiple Frequency Shift Keying (MFSK), and made optimization under transmission time and energy constraint. Cui also considered uncoded MQAM and MFSK in [2]. Abouei et al. analyzed On Off Keying (OOK) besides MQAM and MFSK, demonstrated the simulation distance-based results in [4]. Yet both Cui and Abouei ignored the impact of data link layer, drawing conclusions only at physical layer. In [3], Chen and Gursoy adopted retransmissions in their system and analyzed the energy efficiency of a linear network besides single-hop transmissions, but the retransmission time was unlimited, which is impossible in a practical system. On the other hand, unlimited retransmission time highly restricted the data link layer optimization, and only physical layer optimization was included. In [9], joint routing, media access control (MAC) and data link layer optimization was discussed in sensor networks. However, in their system, retransmission protocol was still not applied.

In this paper, we proposed a new cross-layer optimization design to minimize total energy consumption in a wireless communication system. The system works under a certain packet loss rate constraint, and finite retransmission is allowable. It is proved by simulation results that less energy is consumed compared to physical layer optimization only. 
The rest of this paper is organized as follows. We introduce the system model in Section 2. The analysis of cross-layer design aiming at minimizing the bit energy consumption is presented in Section 3. Section 4 provides several numerical results to confirm the validity of our design. Finally, conclusions are drawn in Section 5.

\section{System Model and Assumptions}

A cross-layer communication system between two wireless nodes is considered, as shown in Figure 1. Data bits from higher layers are processed as packets and stored in a buffer at the transmitter. After modulation, data bits in the packets are mapped into constellation symbols and transmitted from the transmitter to the receiver.

At data link layer, ARQ protocol is employed. When an error occurs in a packet, the ARQ generator at the receiver sends a retransmission request to the ARQ controller via a feedback channel. The packet stored in the buffer will be retransmitted.

At physical layer, MQAM are supported. The modulation mode is chosen by the receiver based on the channel state information (CSI), which is sent back to the transmitter via a feedback channel.

We employed the energy consumption model from [1, $2,9]$. Since there are no complicated blocks such as iterative decoding in the single-antenna system, the power consumed in baseband circuit is much smaller than that in RF circuits, and, for simplifying the model, can be neglected. The system works in three different modes: active mode, transient mode and sleep mode. When transmitting a signal, the system works in active mode. When there is no transmitting, the system works in sleep mode, and the power consumption is much smaller than that in active mode. The system works in transient mode when switching from sleep mode to active mode, whose duration is quite short compared to those of other two modes.

Thus, the total energy consumption can be divided into three parts:

$$
\begin{aligned}
E_{\text {total }} & =P_{o n} T_{o n}+P_{s p} T_{s p}+P_{t r} T_{t r} \\
& =\left(P_{t}+P_{c 0}\right) T_{o n}+P_{s p} T_{s p}+P_{t r} T_{t r}
\end{aligned}
$$

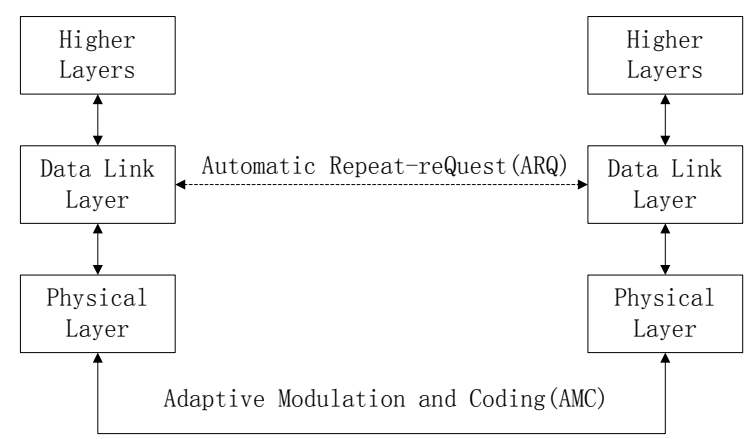

Figure 1. Cross-layer structure. where $P_{o n}, P_{s p}$ and $P_{t r}$ are the power consumption of active mode, sleep mode and transient mode, while $T_{o n}, T_{s p}$ and $T_{t r}$ are the duration of these three modes, respectively. Base on the discussion above, we have $P_{s p} \approx 0$ and $T_{t r} \approx 0 . P_{\text {on }}$ consists of $P_{t}$ and $P_{c 0}$, denoting the power consumption of transmitting signal and circuit, respectively. $P_{c 0}$ is the sum of power consumption of circuits at both transmitter and receiver, including the power amplifier (PA) power consumption $P_{a m p}$, which can be calculated as $P_{a m p}=\alpha P_{t}$, where $\alpha=\xi / \eta-1$ with $\xi$ the peak-to-average ratio(PAR) and $\eta$ the drain efficiency [10]. $\xi=3(\sqrt{M}-1) /(\sqrt{M}+1)$ is a function of MnQAM constellation size.

In this case, $E_{\text {total }}$ can be approximated as

$$
E_{\text {total }} \approx\left(P_{t}+P_{\text {amp }}+P_{c}\right) T_{\text {on }}=\left((1+\alpha) P_{t}+P_{c}\right) T_{\text {on }}
$$

where $P_{c}=P_{c 0}-P_{a m p}$ is the power consumption of all RF circuit blocks except PA. Above, $P_{c}$ is independent of SNR request and transmission distance. On the contrary, $P_{t}$ must guarantee the SNR request for a certain packet loss rate at the receiver after path loss fading.

It is assumed that the transmitted signal is received with additive white Gaussian noise (AWGN) after path loss fading, while perfect CSI is available. Additionally, feedback information is always transmitted correctly and without latency. At the receiver, packet error is detected perfectly, which can be ensured by high reliable cyclic redundancy check (CRC) codes.

\section{Energy Efficiency Analysis}

\subsection{Transmission Power}

Consider the path loss model, the signal power at the receiver, $P_{r}$, is given by

$$
P_{r}(d B)=P_{t}(d B)+K_{d B}-10 \kappa \log _{10}\left(\frac{d}{d_{0}}\right)
$$

where $\kappa$ is the path loss exponent, and $d_{0}$ is the reference distance for the antenna far-field. $K_{d B}$ is calculated as $K_{d B}=20 \log _{10}(\pi) / 4 d_{0}$, where $\lambda$ is the carrier wavelength. At the receiver, the SNR, denoted as $\gamma_{s}$, can be written as

$$
\gamma_{s}=\frac{P_{r}}{N_{0} B}
$$

where $N_{0}$ is the noise power spectrum density and $B$ is the system bandwidth. Take account of AWGN, the MnQAM symbol error rate, marked as $P_{S E}^{n}$, can be derived as

$$
P_{S E}^{n}=2\left(1-\frac{1}{M_{n}}\right) Q\left(\sqrt{\frac{6 \log _{2}\left(\sqrt{M_{n}}\right)}{M_{n}-1} \gamma_{s}}\right)
$$

where $Q(x)=\operatorname{dexp}\left(2 \int_{x}^{\infty} / 2\right)-t^{2} \quad d t$ and $M_{n}=2^{n} \cdot n$ 
is the number of data bits in one MnQAM symbol. Since $P_{S E}^{n}$ for every symbol in a packet is independent, the packet error rate, marked as $P_{P E}^{n}$, is given by

$$
P_{P E}^{n}=1-\left(1-P_{S E}^{n}\right)^{L_{p}}
$$

where $L_{p}$ denotes the number of symbols in one packet. With ARQ protocol, when a transmission error occurs in a packet, corresponding retransmission happens. The maximum allowable retransmission time is denoted as $N_{\max }$, which is determined by the maximum allowable transmission delay. In a realistic system, the maximum retransmission time is finite, which suggested that if a packet is not delivered correctly after $N_{\max }$ retransmissions, it will be dropped. With $P_{P E}^{n}$ and $N_{\max }$, the packet loss rate, $P_{E}^{n}$, can be calculated as

$$
P_{E}^{n}=P_{P E}^{n N_{\max }+1}
$$

Equations (3)-(7) show the relationship between $P_{t}$ and $P_{E}^{n}$. Assume that the max allowable packet loss rate is $P_{E \max }, P_{t}$ should be as small as possible while subjected to

$$
P_{E}^{n} \leq P_{\text {Emax }}
$$

\subsection{Average Transmitting Time}

With $n$ and $L_{p}$, we can see that each packet contains $n$ $L_{p}$ data bits. Assume that there are $L$ data bits need to be transmitted, the total number of successfully transmitted MnQAM packets can be expressed as

$\left\lceil L / n\left(L_{p}-L_{r}\right)\right\rceil$, where $L_{r}$ is the number of redundant symbols in one packet and $\lceil x\rceil$ represents the largest integer less than $x$.

In this case, the average transmission time per packet is

$$
\begin{aligned}
N_{t}^{n} & =\sum_{k=1}^{N_{\max }} k\left(1-P_{P E}^{n}\right) P_{P E}^{n k-1}+\left(N_{\max }+1\right) P_{P E}^{n N_{\max }} \\
& =\frac{1-P_{P E}^{n N_{\max }+1}}{1-P_{P E}^{n}}
\end{aligned}
$$

Clearly, $N_{t}^{n}$ is a function of $P_{P E}^{n}$ and $N_{\max }$. Combing $N_{t}^{n}$ and $\left[L / n\left(L_{p}-L_{r}\right)\right]$, the average number of total transmitting packets can be obtained as

$$
N_{\text {total }}^{n}=\left\lceil\frac{L}{n\left(L_{p}-L_{r}\right)}\right\rceil N_{t}^{n}=\left\lceil\frac{L}{n\left(L_{p}-L_{r}\right)}\right\rceil \frac{1-P_{P E}^{n N_{\max }+1}}{1-P_{P E}^{n}} .
$$

The transmitting time, $T_{\text {on }}^{n}$, is linear with $N_{\text {total }}^{n}$ and is given by

$$
T_{\text {on }}^{n}=N_{\text {total }}^{n} L_{p} T_{s}
$$

where $T_{s}$ is the symbol period. Base on Equations (8)(11), $T_{o n}^{n}$ can be calculated as total active mode time in the cross-layer system.

\subsection{Energy Efficiency}

According to the discussion above, $E_{\text {total }}^{n}$ can be calculated based on Equations (2), (3), and (11). Thus the energy consumption of each successfully delivered data bits, also regarded as energy efficiency, is derived as

$$
E_{b}^{n}=\frac{E_{\text {total }}^{n}}{\left(1-P_{E}^{n}\right) L} .
$$

Therefore, energy efficiency of different constellation sizes can be obtained while the performance and delay requirement are satisfied. By employing AMC at physical layer and ARQ at link layer, energy consumption can be minimized in all distance.

\section{Numerical Results}

To verify the advantage of cross-layer optimization, numerical results are presented in this section. We adopt the circuit energy consumption values in [2]. The bit energy, as shown in Section 3, consists of transmission, retransmission and circuit energy at both transmitter and receiver side under the constraints of $P_{E \max }$ and $N_{\max }$. Parameter settings used in simulations are listed in Table $\mathbf{1 .}$

Figure 2 shows the bit energy consumption of different constellation sizes in a distance ranges from $1 \mathrm{~m}$ to $200 \mathrm{~m}$. In addition, $P_{\text {Emax }}$ is set to be $10^{-3}$ and $N_{\max }$ is 4 . It can be seen that larger constellation sizes cost less energy when $d=1 \mathrm{~m}$. This saving gets smaller when $d$ becomes

Table 1. System parameters.

\begin{tabular}{cc}
\hline$L=106,000$ bits & $\eta=0.35$ \\
$L_{p}=1080$ & $\kappa=3.5$ \\
$L_{r}=20$ & $N_{0}=-120 \mathrm{dBm} / \mathrm{Hz}$ \\
$B=10 \mathrm{kHz}$ & $d 0=1 \mathrm{~m}$ \\
$F_{c}=2 \times 10^{9} \mathrm{~Hz}$ & $P C=210.6 \mathrm{~mW}$ \\
\hline
\end{tabular}

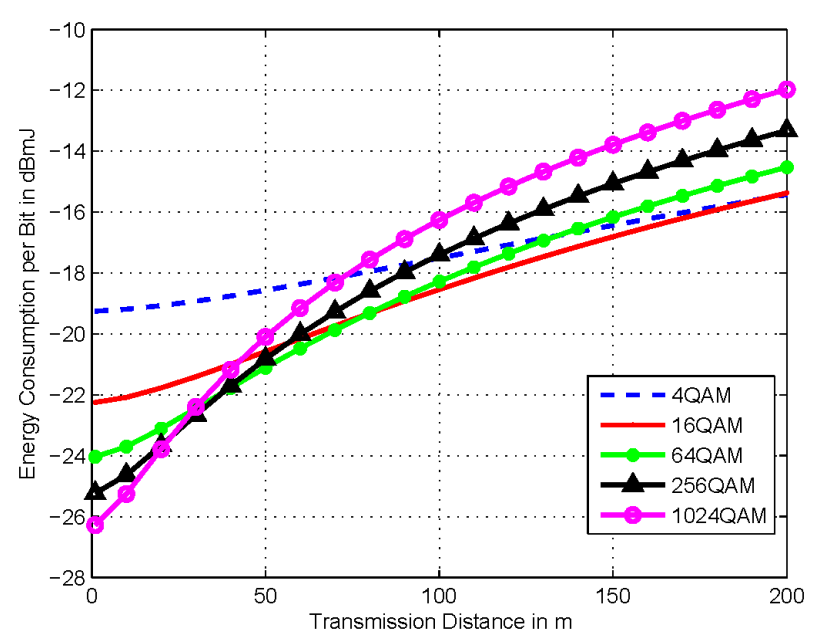

Figure 2. Energy Consumption per Bit under MnQAM with $N_{\text {max }}=4$ and $P_{\text {Emax }}=10^{-3}$. 
larger. And finally, smaller constellation sizes turn out to be more energy efficient when $d=200 \mathrm{~m}$. Generally, larger constellation sizes require higher $P_{t}$ to satisfy the $P_{\text {Emax }}$ constraint. However, $P_{t}$ is small in short distance, in other words, $P_{c}$ dominates the total power consumption. In this case, larger constellation sizes significantly shorten transmission time and reduce the bit energy consumption. On the contrary, $P_{t}$ rises and dominates the total power consumption as $d$ increases, leading to the result that smaller constellation sizes perform better than larger ones. This is similar to former research results derived in no-retransmission cases.

By appropriately designing the AMC, the low-bond bit energy consumption of all constellation sizes, marked as $E_{b \min }$, can be reached. For instance, we choose 1024QAM when $d$ is smaller than $20 \mathrm{~m}$, while 16QAM is applied when $d$ ranges from $80 \mathrm{~m}$ to $180 \mathrm{~m}$.

Figures 3 and 4 illustrate the impact of ARQ employment. In Figure 3, the most energy efficient $N_{\max }$ is 1 when $P_{E \max }$ is $10^{-3}$. However, with a stricter $P_{E \max }$ constraint, $10^{-7}$, larger $N_{\max }$ leads to smaller $E_{\text {bmin }}$, as shown in Figure 4. This is due to the fact that larger $N_{\max }$ reduces $P_{t}$ requirement, but inevitably increases $T_{o n}^{n}$ at the same time. Under a looser constraint $P_{\text {Emax }}$, the impact of $T_{\text {on }}^{n}$ dominates and smaller $N_{\max }$ makes better trade-off. When $P_{E \max }$ constraint becomes stronger, the impact of $P_{t}$ is dominant, and larger $N_{\max }$ performs better.

The largest energy saving achieved by ARQ changes from about $0.5 \mathrm{dBmJ}$ to $0.8 \mathrm{dBmJ}$ when $P_{E \max }$ varies from $10^{-3}$ to $10^{-7}$. We can also observe that $E_{\text {bmin }}$ of $N_{\max }=4$ is larger than that of no-retransmission with PEmax $=10^{-3}$, which emphasizes the importance of ARQ design. With proper $N_{\max }$ setting we can acquire energy saving, while improper $N_{\max }$ may cause lower energy efficiency.

\section{Conclusion}

In this paper, a cross-layer scheme combining AMC and

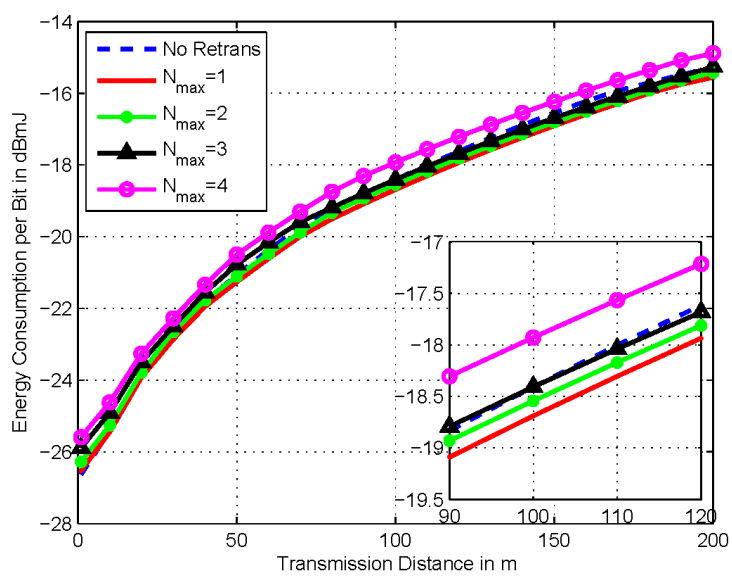

Figure 3. Energy Consumption per Bit under Different Retransmission Time with $P_{E \max }=10^{-3}$.

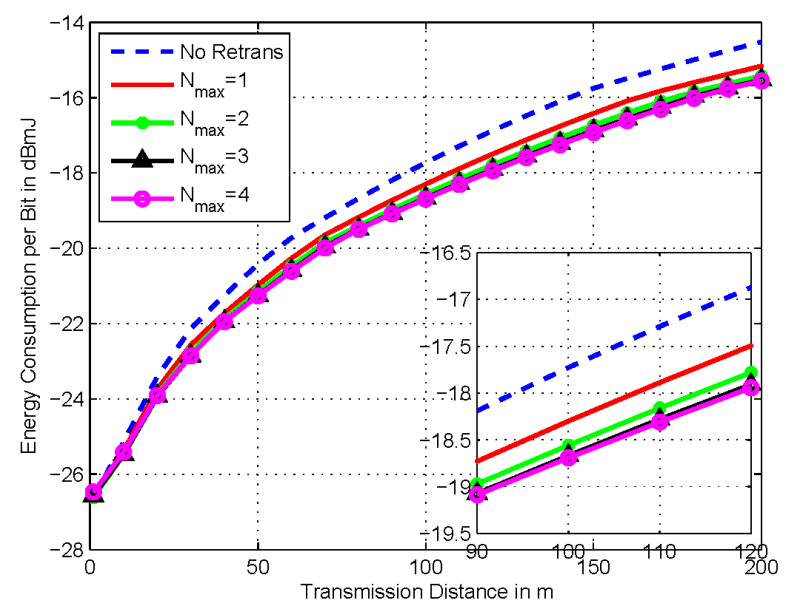

Figure 4. Energy Consumption per Bit under Different Retransmission Time with $P_{E \max }=10^{-7}$.

ARQ is proposed in order to minimize the bit energy consumption under certain delay and performance constraints. We analyzed the energy efficiency of the system, considering dynamic transmission power and duration. Numerical results are presented to verify the validity of the cross-layer design, which brings a significant energy consumption improvement. More channel model and other retransmission protocol will be included in future work.

\section{Acknowledgements}

This work is supported by 863 National Science and Technology Project of the Ministry of Science and Technology of China, Number: SS2012AA011701.

\section{REFERENCES}

[1] S. Cui, A. J. Goldsmith and A. Bahai, "Energy-Constrained Modulation Optimization for Coded Systems," IEEE Global Telecommunications Conference, Vol. 1. 2003, pp. 372-376.

[2] S. G. Cui, "Energy-Constrained Modulation Optimization," IEEE Transactions on Wireless Communications, Vol. 4, No. 5, 2005, pp. 2349-2360. http://dx.doi.org/10.1109/TWC.2005.853882

[3] Q. Chen and M. C. Gursoy, "Energy-Efficient Modulation Design for Reliable Communication in Wireless Networks,” IEEE 43rd Annual Conference on Information Sciences and Systems, 2009, pp. 811-816.

[4] J. Abouei, K. N. Plataniotis and S. Pasupathy, "Green Modulations in Energy-Constrained Wireless Sensor Networks,” IET Communications, Vol. 5, No. 2, 2011, pp. 240-251.

[5] Q. Liu, S. Zhou and G. B. Giannakis, "Cross-Layer Combining of Adaptive Modulation and Coding with Truncated Arq over Wireless Links," IEEE Transactions on Wireless Communications, Vol. 3, No. 5, 2004, pp. 17461755. http://dx.doi.org/10.1109/TWC.2004.833474

[6] S. G. Cui, "Queuing With Adaptive Modulation And 
Coding Over Wireless Links: Cross-Layer Analysis and Design," IEEE Transactions on Wireless Communications, Vol. 4, No. 3, 2005, pp. 1142-1153. http://dx.doi.org/10.1109/TWC.2005.847005

[7] J. Ramis and G. Femenias, "Cross-Layer Design of Adaptive Multirate Wireless Networks Using Truncated HARQ," IEEE Transactions on Vehicular Technology, Vol. 60, No. 3, 2011, pp. 944-954. http://dx.doi.org/10.1109/TVT.2011.2108324

[8] P. Zhang, Y. Miao and Y. Zhao, “Cross-Layer Design of Amc and Truncated Harq Using Dynamic Switching
Thresholds," 2013 IEEE Wireless Communications and Networking Conference (WCNC), 2013.

[9] S. Cui, R. Madan, A. Goldsmith and S. Lall, "Joint Routing, Mac, and Link Layer Optimization in Sensor Networks with Energy Constraints,” 2005 IEEE International Conference on Communications, Vol. 2, 2005, pp. 725-729.

[10] T. H. Lee, "The Design of CMOS Radio-Frequency Integrated Circuits,” Cambridge University Press, Cambridge, 2004. 